\title{
Morfometría de la copa de Araucaria angustifolia en sitios naturales en el sur de Brasil
}

\author{
Morphometry of the crown of Araucaria angustifolia in natural sites in southern Brazil
}

\author{
André Felipe Hess ${ }^{\text {a*}}$, Táscilla Loiola ${ }^{\text {b }}$, Isadora Arruda de Souza ${ }^{\text {b }}$, Bruno Nascimento ${ }^{\text {b }}$ \\ *Autor de correspondencia: ${ }^{a}$ Universidad del Estado de Santa Catarina, Centro de Ciencias Agroveterinárias, \\ Departamento de Ingeniería Forestal, Av. Luiz de Camões 2090, CEP 88520-000, Lages, SC, Brasil, \\ tel.: 55-49-32899255, hessandre@yahoo.com.br \\ ${ }^{\mathrm{b}}$ Universidad del Estado de Santa Catarina, Centro de Ciencias Agroveterinárias, Departamento de Ingeniería Forestal, \\ Lages, SC, Brasil.
}

\begin{abstract}
SUMMARY
In forest management, it is necessary to understand the morphometric relationships to provide information about the characteristics of the morphological adjustment of the crown caused by competition for environmental resources in forest communities. In this study, we analyzed the generated morphometric characteristics with inter-dimensional equation fit for individual trees of Araucaria angustifolia in different sites of the Araucaria Forest in Southern Brazil, checking if there are different morphological responses of the crown according to different stem dimensions. To do this, samples were taken from 186 trees in a single-tree based approach on the extent of forest diameter distribution; dendrometric and morphometric variables were measured. The increase in diameter was obtained through the chronology of ring widths. The adjustment results in mixed models show that the species differs in the proportion of the crown among sites. The equations have been able to explain the relation between form and size. Changes in morphology are related to competition and changes in diameter at breast height, which indicates that forest management should be based on morphometric differences to propose silvicultural interventions in the forest, thus resulting in a positive way in increasing the growth rates of individual trees.
\end{abstract}

Key words: morphometry of the crown, competition, growth, modeling.

\section{RESUMEN}

En el manejo forestal es necesario comprender las relaciones morfométricas para proporcionar información sobre las características del ajuste de la morfología de la copa causadas por la competencia, por los recursos ambientales en las comunidades forestales. En este estudio, se analizaron las características morfométricas generados con ajuste de ecuación inter-dimensional para los árboles individuales de Araucaria angustifolia en diferentes sitios del Bosque de Araucaria en el sur de Brasil, para comprobar si hay diferentes respuestas morfológicas en la copa de acuerdo a las diferentes dimensiones del fuste. Para ello, se tomaron muestras de 186 árboles en un enfoque basado en árbol individual en la amplitud de distribución de diámetro del bosque y se midieron sus variables dendrométricas, morfométricas y medidas del incremento en diámetro a través de la cronología de anchos de anillos. Los resultados del ajuste de los modelos mixtos muestran que la especie difiere en la proporción de copa entre los sitios. Las ecuaciones fueron capaces de explicar la relación entre forma y dimensión y las variaciones en la morfometría están relacionadas con la competencia y cambios en el diámetro a la altura do pecho, lo que indica que el manejo forestal debe basarse en la diferencias morfométricas para proponer intervenciones silvícolas en el bosque, lo que resulta de manera positiva en aumento de las tasas de crecimiento de los árboles individuales.

Palabras clave: morfometría de la copa, competencia, crecimiento, modelaje.

\section{INTRODUCCIÓN}

Araucaria angustifolia (Bertol.) Kuntze (araucaria) es un árbol del Bosque de Araucaria en América del Sur. La araucaria es una especie pionera, intolerante a la sombra y domina el estrato superior del dosel. Originalmente, los bosques de araucarias ocuparon $177.600 \mathrm{~km}^{2}$ en Brasil y en Argentina $2.100 \mathrm{~km}^{2}$ (Giraudo et al. 2003) en un intervalo que incluye las coordenadas de $19^{\circ} 15^{\prime}-31^{\circ} 30^{\prime} \mathrm{S}$ y $41^{\circ} 30^{\prime}$ - 54²30' $\mathrm{O}$ y altitudes de 200 a $1.800 \mathrm{~m}$.
La mayoría de los remanentes de este tipo de bosque están sin manejo e intervenciones silviculturales cerca de 40 años debido, específicamente, a la legislación restrictiva, con alegación de pérdida de la diversidad genética y la falta de investigación sobre el uso sostenible de la especie. Stefenon et al. (2008) argumentan que el moderado a alto nivel de diversidad genética en las poblaciones retenidas de Araucaria angustifolia, después de una intensa fragmentación del bosque natural, tiene el potencial para producir materiales con suficiente diversidad genética para 
la conservación de la especie, a través de la creación de bosques plantados o la gestión sostenible de los bosques remanentes existentes.

La prohibición de manejo y el uso de la especie en Brasil impide que los bosques sean utilizados como una fuente de renta, mientras que bloquea las iniciativas de manejo sostenible, que en sentido amplio, incluye la recuperación y conservación a largo plazo (Diez 2012), bien como una reducción en las tasas de crecimiento (por lo tanto, la reducción en la absorción de carbono), la competencia, menor regeneración, la pérdida de productividad y la rentabilidad (Hess et al. 2014). Sin embargo, se da la oportunidad de investigar y obtener información sobre las relaciones morfométricas, tipo de competencia (simétrica, asimétrica), estudios de dendrocronología, conocer el incremento de las variables dendrométricas, dinámica estructural, etc., con el objetivo de ayudar en el cambio de la ley para liberar el manejo forestal de la especie, mantener su conservación y la perpetuidad de los remanentes.

Minatti (2015), ajustando ecuaciones del incremento de araucaria en tres regiones del sur de Brasil en función del diámetro a la altura del pecho y de la edad, encontró coeficiente angular negativo, lo que indica que la especie ha llegado a su capacidad de crecimiento y los bosques con araucaria están en estancamiento. En su estudio encontró un incremento medio anual en diámetro que varió desde 0,3 hasta $0,6 \mathrm{~cm}$ año ${ }^{-1} \mathrm{y}$, que los árboles de menor diámetro están creciendo menos que el diámetro medio de todas las clases, lo que indica la necesidad de intervenciones de raleo. Así como, que están en competencia por espacio y recursos, siendo importante conocer los índices morfométricos y sus relaciones con la dimensión para ayudar en intervenciones de raleo y para el manejo sostenible.

McIntosh et al. (2012) dicen que el uso de los datos de forma de la copa puede ayudar en monitoreo forestal, además de informar sobre la zona de influencia de la copa (superposición) y el grado de competencia del árbol. Leite et al. (2012) señalan que las dimensiones de la copa interfieren en el logro de procesos fisiológicos $\mathrm{y}$, a menudo, estos se utilizan como indicadores de la capacidad de un árbol de competir por los recursos. Características tales como la extensión, diámetro y volumen de la copa sirven como indicadores de respiración fotosintética y la capacidad de transpiración (Sprinz y Burkhart 1987).

Lang et al. (2010) dicen que en los bosques la luz es ampliamente reconocida como uno de los factores determinantes del crecimiento y uno de los ejemplos más importantes de un recurso que genera competencia dimensión-asimétrica (competencia por recurso encima del nivel del suelo y que conduce a una mayor desigualdad de la biomasa dentro de una población, concluyendo que el desarrollo en desigualdad de dimensión es prueba de la competencia asimétrica), lo que sugiere que las respuestas morfológicas tales como, dimensión de la copa y la arquitectura del copa pueden ser el resultado de la competencia por la luz.
En el presente estudio se asume que los índices morfométricos y sus variaciones reflejan el efecto de la competencia en el pasado (competencia en la que el árbol fue sometido y que contribuyó con las dimensiones de las copas presentadas actualmente), provocando cambios ínter dimensionales en el árbol, que se pueden expresar mediante funciones de regresión, y que esas variaciones son también de la captación de recursos para el crecimiento, según lo propuesto por Cunha y Finger (2013). Así, para dos árboles del mismo diámetro se ha supuesto que muestra un considerable grado de competencia el árbol con menor diámetro de copa. Por lo tanto, la reducción en la tasa de crecimiento puede proceder de la reducción en el tamaño de la copa como resultado del efecto de la competencia en años anteriores.

Cunha y Finger (2013) consideraron que el ajuste de ecuaciones lineales por ser expresiones matemáticas simples no representan necesariamente todo el proceso ontogenético de la competencia y los cambios dimensionales de las árboles. Sin embargo, utilizar solamente la densidad como una medida de la competencia para evaluar el desempeño de árboles en bosques mixtos puede generar modelos sesgados. No obstante, los resultados y su información contribuyen para ayudar en los planes de manejo de la especie.

Dada la importancia histórica y económica de la especie, el riesgo de perpetuidad y conservación de los bosques de araucaria debido a la falta de manejo, se plantean las preguntas: 1) ¿Cuál es el nivel de competencia en el bosque de araucaria? 2) ¿La competencia provoca cambios en la morfometría de la copa en relación con diferentes diámetros a la altura del pecho? y 3) ¿Evaluar los índices morfométricos y predecir sus relaciones puede servir como un subsidio a las intervenciones sostenibles y así promocionar los cambios en la legislación forestal en el Sur de Brasil? Se plantean dos hipótesis: 1) hay variaciones en los índices morfométricos y en la morfología de la copa en relación a su dimensión en los diferentes sitios de su ocurrencia y 2) el ajuste de modelos lineales mixtos puede ser empleado para explicar esta variación.

Por lo tanto, el objetivo de este estudio es calcular los índices morfométricos para conocer sus variaciones de acuerdo con sus dimensiones en los remanentes de los bosques de araucaria y ajustar mediante el uso de modelo lineal mixto ecuaciones de relación de la proporción de copa $(p c \%)$ en función del diámetro a la altura del pecho, con el fin de responder las preguntas y ayudar en el uso sostenible de la especie.

\section{MÉTODOS}

Área de estudio. El estudio se realizó en tres localidades con Bosque de Araucaria en el sur de Brasil, São Joaquim (SJQ), Urupema (URU) y Painel (PNL), con origen natural de Araucaria angustifolia, especie de hoja perenne, con copa variando en forma de cono a hemisférica y la forma madura de umbela (Seitz 1986). En cada local, se 
seleccionaron sitios de estudio con remanentes de Bosque de Araucaria. El sitio SJQ, con coordenadas (UTM) $22 \mathrm{~S}$, $600.418 \mathrm{~m} \mathrm{~S}$ y $6.879 .092 \mathrm{~m} \mathrm{O}$, área total de 73,46 ha y 1,86 ha de área muestreada; URU coordenadas 595.808 $\mathrm{m} \mathrm{S}$ y $6.910 .762 \mathrm{~m} \mathrm{O}, 18,08$ ha de área total y 1,2 ha de área muestreada y PNL con coordenadas $610.183 \mathrm{~m} \mathrm{~S}$ y 6.906.780 m O, con una superficie total de 50,47 ha y área de muestra de 1,3 ha.

La región se caracteriza por tener clima $\mathrm{Cfb}$, según clasificación de Köppen, clima templado, constantemente húmedo sin estación seca. En São Joaquim, la altitud es de $1.166 \mathrm{~m}$, temperatura media anual de $14{ }^{\circ} \mathrm{C}$ y precipitaciones de $1.740 \mathrm{~mm}$, en Urupema la altitud es de $1.259 \mathrm{~m}$, $13,7^{\circ} \mathrm{C}$ de temperatura media anual y $1.722 \mathrm{~mm}$ y Painel con $1.123 \mathrm{~m}$ de altitud, $15,3{ }^{\circ} \mathrm{C}$ y $1.543 \mathrm{~mm}$ de precipitación anual (Álvarez et al. 2013).

Los suelos en São Joaquim son de la asociación Neossolo Litólico + Cambissolos Hápicos + Neossolos Litólicos Eutróficos, con relieve ondulado fuerte. En Urupema los suelos se clasifican como asociación Cambissolo Húmico + Neossolo Litólico, arcillosos, fase pedregosa, relieve ondulado (sustrato efusivo de la formación Serra Geral) + Nitossolo Bruno distrófica. En Painel los suelos son Noessolos Litílicos Distroúmibricos, arcillosos, fase rocosa (sustrato efusiva de la formación Serra Geral) + Cambissolos Háplicos, con relieve suavemente ondulado (EMBRAPA 2012).

Toma de datos. Fueron muestreados 186 árboles en un enfoque basado en árbol individual en la amplitud de distribución de diámetro en el Bosque de Araucaria. El cálculo de la suficiencia de muestras señaló la necesidad de $\mathrm{n}=36$ árboles en SJQ, $\mathrm{n}=25$ en URU y $\mathrm{n}=35$ en PNL (total $\mathrm{n}=$ 96), lo que indica que el levantamiento de 186 árboles fue suficiente para los sitios de estudio. Los remanentes forestales tienen densidad que varía de menos de 200 a más de 300 árboles por hectárea (Hess et al. 2014).

En cada árbol, en cada sitio, fue medido el diámetro a la altura del pecho $(d)$, altura de inserción de la copa (hic), definida como la altura desde el suelo a la inserción de la copa viva, la altura total $(h t)$, también la medición de cuatro rayos de la copa en las direcciones cardinales (Norte, Sur, Este y Oeste), usando el aparato TruPulse 200 y una brújula, siendo cuatro rayos suficiente para la especie araucaria, pues presenta copa simétrica (Costa et al. 2013).

Con las medidas se obtuvieron:

$$
\begin{gathered}
\text { radio medio de la copa, } \overline{r c}=\sum_{i=1}^{n=4} \frac{r c}{4} \\
\text { diámetro de la copa, } d c=2 * \overline{r c} \\
\text { área de proyección de la copa, } A p=\pi * \overline{r c}^{2} \\
\text { extensión de la copa, } e c=h t-h i c
\end{gathered}
$$

$$
\begin{gathered}
\text { grado de esbeltez, }(h t / d) \\
\text { índice saliente, }(d c / d) \\
\text { índice de amplitud, }(d c / h t) \\
\text { formal de copa, }(d c / e c)
\end{gathered}
$$

$$
\text { proporción de la copa, } p c \%=\left(\frac{e c}{h t}\right) * 100
$$

Fueron extraídos de cada árbol dos tarugos de incremento perpendicularmente al nivel del diámetro a la altura del pecho (d) (Asmann 1970). Los tarugos fueron pegados en soportes de madera, pulidos y marcados sus anillos. Se midieron la anchura entre los anillos permitiendo la determinación del incremento anual en diámetro $(d)$, usando el dispositivo de medición digital Lintab-6 con una precisión de $0,01 \mathrm{~mm}$ con soporte del software Time Series Analyses (TSAP - Win), con procedimiento de datación cruzada.

De los individuos de cada sitio fue considerado el árbol de mayor diámetro (objetivo) y el diámetro de los individuos cercanos para caracterizar la competencia. Se calculó el índice de competencia (IC) sin tener en cuenta la distancia como propuesto por Daniels et al. (1986):

$$
\mathrm{IC}_{\mathrm{j}}=d_{\mathrm{j}}^{2} * n / \sum_{\mathrm{i}} d_{\mathrm{i}}^{2}
$$

donde $d_{\mathrm{j}}$ : diámetro de la árbol objetivo, $d_{\mathrm{i}}$ : diámetro del competidor, $n$ : número de árboles competidores. Cuanto mayor sea el IC, mayor capacidad competitiva del árbol objetivo respecto a sus vecinos.

Los datos de morfometría y los índices morfométricos fueron sometidos a análisis de correlación de Pearson para identificar los que tienen mayor correlación y así ayudar en la interpretación de las condiciones de árboles de araucaria creciendo en bosques naturales. Sólo se consideraron correlaciones con valor mayor o igual a 0,4 .

Análisis estadístico. Los análisis estadísticos fueron procesados utilizando el SAS 9.3 (Statistical Analysis System). Se probó la diferencia de la variable dependiente proporción de copa $(p c \%)$ mediante el ajuste de modelo lineal mixto con el diámetro como una variable independiente (explicativa). La inclusión de efectos aleatorios en la variable explicativa diámetro representa la heterogeneidad natural dentro y entre individuos debido a factores genéticos y ambientales (Schneider et al. 2009) que se puede observar con la modificación de las dimensiones diametrales. Por lo tanto, con la inclusión de efectos aleatorios se ajusta el modelo por factores no medidos y se obtienen estimaciones más precisas. Fue comprobado el efecto aleatorio en nivel (el origen) y la inclinación (slope) con los datos procedentes de los tres sitios de estudio. 
La precisión del modelo se evaluó por el criterio de información de Akaike (CIA), criterio de información baynesiano (CIB) y análisis gráfico de los residuos. Para la interpretación de los resultados, cuanto menor los valores de ambos criterios (CIA) y (CIB), mejor ajuste del modelo. Los residuos del modelo no mostraron violación de las condiciones de regresión (normalidad y homogeneidad de varianza).

\section{RESULTADOS}

Dimensión y morfometría. Con los datos muestreados se obtuvo la distribución de árboles por clase de diámetro (figura 1). El mayor número de árboles está en las clases de 25 a $40 \mathrm{~cm}$ de diámetro. El sitio SJQ fue el que presentó el mayor número de árboles en clases de mayor diámetro.

Con los datos se obtuvieron los resultados de los índices morfométricos y de incremento (cuadro 1) y, posteriormente, el ajuste de las ecuaciones de los modelos lineales mixtos para la proporción de la copa por sitio de estudio (figura 2).

La altura de inserción de la copa (hic) presentó valores entre 3,5 m y 22,9 m. La extensión de la copa varió entre 0,3 y $13,1 \mathrm{~m}$, promedio de $5,1 \mathrm{~m}$ en SJQ, 5,3 $\mathrm{m}$ en URU y 6,3 en PNL. El diámetro de la copa varió entre 1,3 y $16,6 \mathrm{~m}$, siendo que los valores más bajos puede indicar menos espacio lateral para el crecimiento y desarrollo de los árboles, o árboles jóvenes, lo que explica menor área

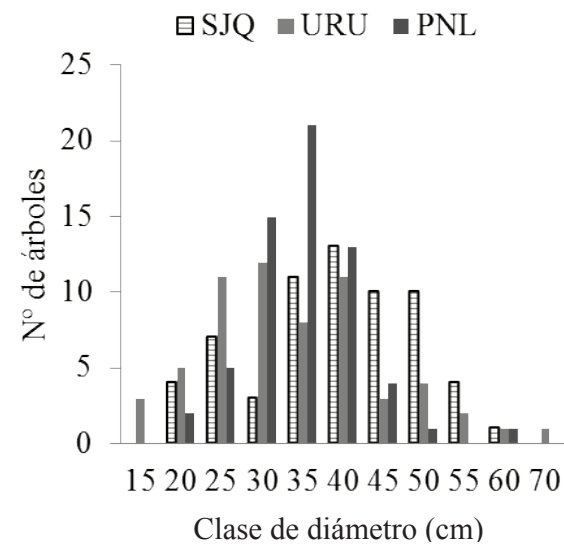

Figura 1. Estructura diamétrica por sitio de estudio y el número de árboles individuales por clase de diámetro.

Diametric structure by study site and the number of individual trees by diameter class.

de proyección de la copa en sitio PNL. Los bajos valores de la desviación estándar para los índices indica que los datos se encuentran cerca de la media.

El espacio promedio ocupado por árboles (área de proyección de copa) fue de $80,6 \mathrm{~m}^{2}$ en SJQ, 56,4 $\mathrm{m}^{2}$ en URU y $20,2 \mathrm{~m}^{2}$ en PNL, siendo la variación explicada por la amplitud dimensional de los individuos, sitio y densidad. Estos

Cuadro 1. Características dimensionales e índices morfométricos en los tres sitios de estudio de Araucaria angustifolia en el sur de Brasil (n: 186).

Dimensional characteristics and morphometric indices in the three study sites for Araucaria angustifolia in southern Brazil.

\begin{tabular}{lcccccccccccc}
\hline \multicolumn{1}{c}{ Sitio SJQ } & $d$ & $h t$ & $h i c$ & $d c$ & $a p$ & $e c$ & $p c$ & $g e$ & $i s$ & $i a$ & $f c$ & $i d$ \\
\hline Médio & 41,4 & 17,0 & 11,9 & 9,7 & 80,6 & 5,1 & 30,1 & 43,4 & 23,6 & 0,5 & 2,3 & 0,4 \\
Máximo & 60,2 & 26,1 & 22,9 & 16,6 & 216,4 & 10,9 & 63,4 & 77,1 & 30,9 & 1,2 & 6,1 & 0,7 \\
Mínimo & 20,1 & 11,9 & 5,3 & 4,2 & 13,9 & 1,6 & 6,5 & 24,1 & 16,7 & 0,2 & 0,7 & 0,2 \\
$\sigma$ & 9,8 & 2,7 & 2,8 & 2,7 & 42,0 & 1,9 & 10,8 & 12,1 & 3,4 & 0,2 & 1,3 & 0,3 \\
Sitio URU & & & & & & & & & & & & \\
\hline Médio & 36,6 & 14,2 & 8,9 & 8,1 & 56,4 & 5,3 & 37,5 & 41,7 & 22,9 & 0,6 & 2,5 & 0,8 \\
Máximo & 70,0 & 23,0 & 19,2 & 13,5 & 142,1 & 13,1 & 66,9 & 82,7 & 31,4 & 0,9 & 38,7 & 1,4 \\
Mínimo & 18,8 & 8,8 & 4,4 & 4,1 & 13,2 & 0,3 & 2,3 & 19,9 & 10,5 & 0,3 & 0,8 & 0,4 \\
$\sigma$ & 11,0 & 2,9 & 2,9 & 2,2 & 30,3 & 2,3 & 14,4 & 12,9 & 4,2 & 0,2 & 4,9 & 0,4 \\
\multicolumn{1}{c}{ Sitio PNL } & & & & & & & & & & & & \\
\hline Médio & 37,5 & 14,4 & 8,0 & 4,8 & 20,2 & 6,3 & 43,5 & 39,1 & 12,8 & 0,3 & 0,9 & 0,9 \\
Máximo & 62,7 & 19,0 & 11,5 & 8,8 & 60,8 & 12,0 & 72,7 & 55,9 & 22,0 & 0,7 & 4,6 & 1,8 \\
Mínimo & 23,6 & 11,0 & 3,5 & 1,3 & 1,2 & 1,0 & 8,8 & 26,2 & 3,5 & 0,1 & 0,2 & 0,4 \\
$\sigma$ & 6,7 & 1,9 & 1,9 & 1,7 & 14,3 & 2,3 & 13,4 & 7,0 & 4,1 & 0,1 & 0,6 & 0,5 \\
\hline
\end{tabular}

Donde: $d$ : diámetro a la altura del pecho $(\mathrm{cm})$; $h t$ : altura total $(\mathrm{m})$; hic: altura de inserción de la copa $(\mathrm{m})$; $d c$ : diámetro de copa $(\mathrm{m})$; ap: área de proyección de copa $\left(\mathrm{m}^{2}\right) ;$ ec: extensión de la copa $(\mathrm{m}) ; p c$ : proporción de copa (\%); ge: grado de esbeltez; is: índice saliente; ia: índice de amplitud; $f c$ : formal de copa; id: incremento en diámetro $(\mathrm{cm})$; $\sigma$ : desviación estándar. 


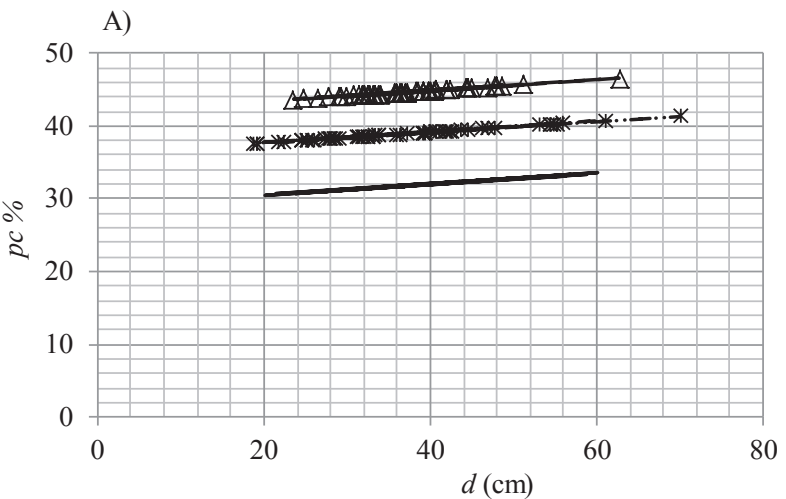

$\longrightarrow$ pc\% est. SJQ - *- pc\% est. URU $\rightarrow-\mathrm{pc} \%$ est. PNL

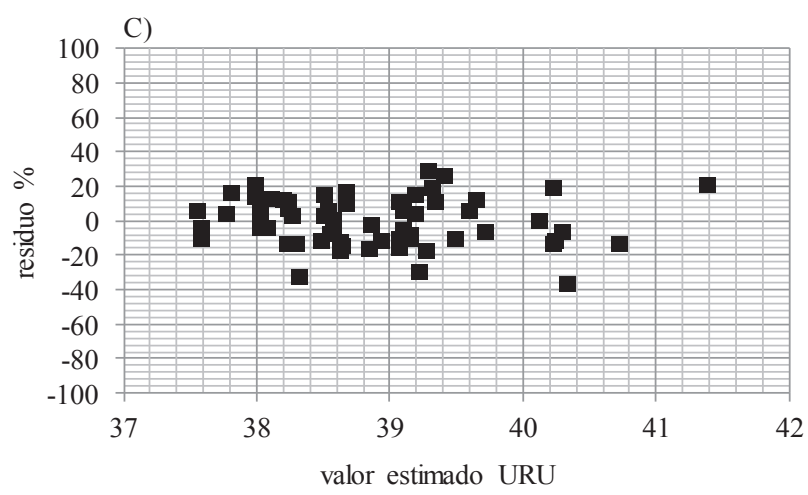

B)

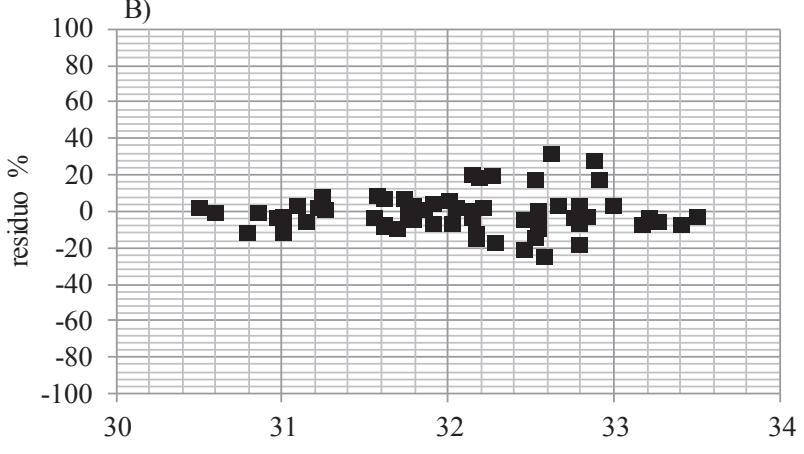

valor estimado SJQ

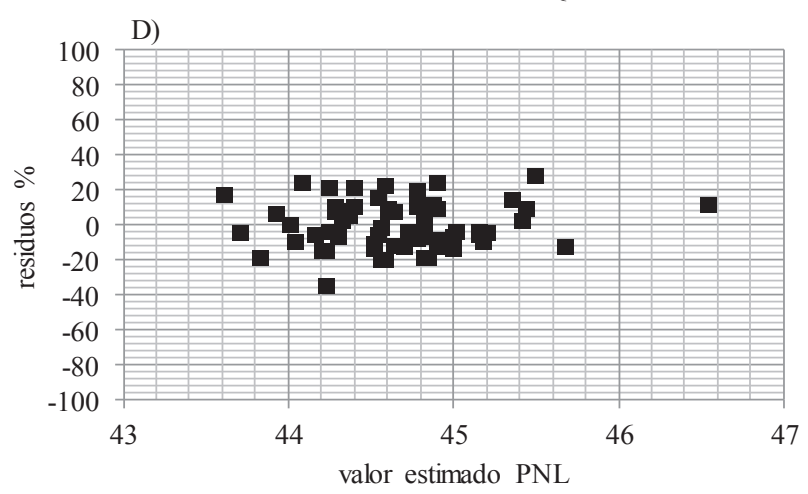

Figura 2. A) Ajuste do modelo lineal mixto para la proporción de copa ( $p c \%)$ en función del diámetro a altura do pecho $(d)$ de Araucaria angustifolia en los tres sitios de estudio en el sur de Brasil donde hubo el efecto aleatorio de nivel $\left(\mathrm{b}_{0}\right)$ y no de inclinación, donde: pc \% est. SJQ, URU y PNL: proporción de copa estimada a São Joaquim, Urupema y el Painel. (B), (C) y (D) análisis gráfico de los residuales del modelo lineal mixto por sitio de estudio.

A) Adjustment of the mixed linear model for proportion of the crown ( $p c \%$ ) depending on the diameter (D) for Araucaria angustifolia in the three study sites where there was the random effect level (intercept) and not tilt effect, where: $p c \%$ est. SJQ, URU and PNL: proportion of the crown estimated to São Joaquim, Urupema and Painel. (B), (C) and (D) graphical analysis of the residuals of the linear mixed model by study site.

valores indican una densidad ideal de 124 árboles por hectárea en SJQ, 177 en URU y 500 en PNL. Si fueran árboles creciendo libres, mayor sería el área de proyección de copa.

La proporción de copa $(p c \%)$ presentó valores que van desde 2,3 a 72,7 \%. El mayor valor promedio fue para el sitio PNL (43,5\%), lo que indica un bosque con menor diámetro a altura del pecho $(d)$, menor altura de inserción de la copa y mayor extensión de copa, pues según Costa et al. (2016), árboles de mayor $d$ tienden a presentar menor extensión de copa, revelando que la mortalidad de las ramas de la base de la copa fue mayor.

El grado medio de esbeltez fue similar para los sitios (valor $\sim 40$ ) demostrando árboles con menor manto de copa. El índice de amplitud mostró valores promedios entre 0,3 y 0,6 , siendo utilizado como un indicador para raleo. El índice saliente varió de 12,8 a 23,6 que indica que la araucaria tiene un diámetro de la copa en torno de 20 veces el diámetro a la altura del pecho.

El formal de copa $(f c)$ fue menor en el sito PNL, el valor ha indicado que la especie en este sitio presenta copas cónicas y árboles creciendo en altura. SJQ y URU presen- taron árboles con mayor formal de copa, copas con forma elipsoidal, plana o de umbela (Costa et al. 2016).

El sitio de SJQ mostró menor valor de incremento promedio en diámetro y PNL, el mayor valor. Sin embargo, en todos los sitios la tasa de incremento actual ocurre en el $50 \%$ del valor máximo. Los resultados muestran que el sitio SJQ caracteriza un bosque de mayor edad y diámetro y árboles que han alcanzado la capacidad máxima de crecimiento. Los sitios URU y PNL, árboles más jóvenes con incremento promedio mayor, como también las tasas en descenso debido a la competencia por el espacio y los recursos disponibles.

El sitio en SJQ presentó un índice de competencia de 1,9, URU de 2,6 y PNL de 1,6, lo que demuestra que los árboles están en competencia por los recursos ambientales y que esto influye en las características de la forma y dimensión de la copa. El sitio URU tiene árboles competidores de menor diámetro que contribuyeron al mayor valor del índice de competencia. Los resultados muestran que la competencia está relacionada con la extensión y el diámetro de la copa indicando la necesidad de intervenciones silviculturales. 
Los índices morfométricos presentaron correlación entre sí (cuadro 2). Los valores variaron de 0,94 a $-0,42$ y explican los resultados de los índices promedios calculados para el bosque natural. La mayor correlación fue entre $i a$ y $d c$. El índice ec mostró correlación de 0,87 con la $p c \%$, lo que explica árboles de menor tronco libre, pues mayor la extensión de la copa, menor radio de copa, más jóvenes y menor diámetro, como se encuentra en sitio PNL. Ello también puede ser verificado por la correlación negativa entre la $p c \%$ con la altura de inserción de la copa (hic).

El diámetro a la altura del pecho $(d)$ mostró una correlación negativa con ge que indica que cuanto menor es el $d$ mayor valor del ge, lo que demuestra que los árboles crecen más en altura que en $d$, teniendo relación con la estabilidad de los árboles, la competencia y el sitio.

Los resultados de correlación indican que las relaciones establecidas con variables dendrométricas e índices morfométricos explican los índices, la competencia, características del sitio, la dinámica de la forma y crecimiento de los individuos en desarrollo en el bosque.

Ajuste de la forma y dimensión. Los resultados no indican diferencias significativas de efectos fijos en el diámetro $(P>0,05)$. Sin embargo, el efecto aleatorio en el intercepto muestra una diferencia significativa en la proporción de copa para los tres sitios de estudio (figura 2A) con valor de $(P<0,0001)$. Se denota así, para los sitios, diferencias significativas en el nivel (parámetro $b_{0}$ ), pero no se demostró efecto aleatorio de la inclinación (parámetro $b_{1}$ ).

El modelo lineal mixto y su efecto aleatorio para los sitios de estudio para estimar la proporción de copa ( $p c \%)$ en función del diámetro a la altura del pecho $(d)$ se expresó con las ecuaciones:

a) Sitio de São Joaquim (SJQ):

$$
p c \%=35,6782+(-6,6655)+0,07485 * d
$$

b) Sitio de Urupema (URU):

$$
p c \%=35,6782+(0,4845)+0,07485 * d
$$

c) Sitio de Painel (PNL):

$$
\text { pc } \%=35,6782+(6,181)+0,07485 * d
$$

El ajuste del modelo lineal mixto mostró un valor del criterio de información Akaike (CIA) de 1.504,8 y criterio de información bayesiano (CIB) de 1.503,0.

El ajuste mostró una correlación positiva entre las variables, siendo que a medida que aumenta el diámetro aumenta la proporción de copa. Los residuos presentaron homogeneidad de la varianza y ninguna tendencia en el ajuste (figura 2 (B), (C) y (D)), que muestra que las estimaciones de esta relación pueden ser generadas con precisión.

Los sitios de SJQ y URU mostraron valores más bajos para la proporción de copa, resultado del aumento en diámetro, efecto de la competencia en los individuos de pequeño diámetro (lo que demostró también los valores de índice de competencia), reflejado directamente en la disminución de la extensión de copa y, respectivamente, menor área de asimilación fotosintética.

\section{DISCUSIÓN}

Índices morfométricos. Los índices morfométricos (cuadro 1) indican que en los sitios estudiados los árboles sufrieron competencia por el espacio de crecimiento, lo que limita la expansión lateral de la copa y favoreciendo el crecimiento vertical. Sin embargo, el régimen de perturbaciones ayudó al crecimiento de los árboles de mayor dimensión (Pretzsch y Schütze 2005).

SJQ mostró mayor valor para altura de inserción de copa (hic) lo que explica árboles de mayor diámetro a la altura del pecho $(d)$, menor extensión de la copa $(e c)$ y menor valor de la proporción de la copa $(p c \%)$. Ello

\begin{tabular}{|c|c|c|c|c|c|c|c|c|}
\hline Variables & $f_{c}$ & ge & $i a$ & & $p c \%$ & hic & $d c$ & $h t$ \\
\hline$d$ & - & $-0,7^{*}$ & & - & - & - & $0,53 *$ & - \\
\hline$e c$ & $-0,42^{*}$ & - & & - & $0,87^{*}$ & $-0,46^{*}$ & - & - \\
\hline$f_{c}$ & - & - & $0,44 *$ & & $-0,44^{*}$ & - & $0,43^{*}$ & - \\
\hline ge & - & - & $-0,42 *$ & & - & - & - & - \\
\hline is & - & - & $0,83 *$ & & - & - & $0,83^{*}$ & - \\
\hline$i a$ & - & - & - & & - & - & $0,94^{*}$ & - \\
\hline$p c$ & - & - & - & & - & $-0,79^{*}$ & - & - \\
\hline hic & - & - & - & & - & - & - & $0,72 *$ \\
\hline
\end{tabular}

Cuadro 2. Correlación de Pearson para índices morfométricos de Araucaria angustifolia en bosque natural en el sur de Brasil. Pearson correlation for morphometric indices for Araucaria angustifolia in natural forest in southern Brazil.

Donde: $d$ : diámetro a la altura del pecho $(\mathrm{cm}) ; h t$ : altura total $(\mathrm{m})$; hic: altura de inserción de la copa $(\mathrm{m})$; $d c$ : diámetro de copa $(\mathrm{m}) ; a p$ : área de proyección de copa $\left(\mathrm{m}^{2}\right) ; e c$ : extensión de la copa $(\mathrm{m}) ; p c \%$ : proporción de copa (\%); ge: grado de esbeltez; is: índice saliente; ia: índice de amplitud; $f_{c}$ : formal de copa; $(*)$ : probabilidad $(P<0,0001) ;(-)$ : índices con ninguna correlación. 
indica, de acuerdo con las características ontogenéticas de araucaria, árboles que alcanzaran mayor edad, siendo indicador de intervención silvícola en el bosque. Estos valores indican que en el pasado estos árboles salieron de la competencia. De acuerdo con los valores de hic, ec y $p c \%$, URU y PNL son los sitios con los árboles más jóvenes y menores $d$, lo que explica mayor índice de competencia.

$\mathrm{El}$ análisis de estos índices (hic) (ec), explica los menores valores de diámetro de copa, en consecuencia, menor radio de copa y área de proyección de copa en los sitios de URU y PNL, indicando que el aumento del área de proyección de la copa puede ser un indicador, durante el desarrollo de la dinámica de los cambios en la forma de los árboles en el bosque, de intervención silvicultural.

La extensión de la copa $(e c)$ presenta correlación de $-0,46$ con la altura de inserción de copa (hic), indicando que menor $e c$ es un indicativo de mayor fuste utilizable, autorraleo, competencia e inferior proporción de copa, lo que reduce la capacidad fotosintética y el crecimiento.

El valor del índice de proporción de la copa ( $p c \%)$ mostró que en el sitio SJQ los árboles tienen mayor $d$ y menor valor para el índice, indicando reducción en el crecimiento en altura, pues alcanzaran el dosel superior, mayor edad y mayor tronco utilizable, indicado por una correlación negativa con la altura de inserción de la copa y positiva con la extensión de la copa.

La amplitud de variación del índice, muestra asociación con los factores bióticos y abióticos (Lang et al. 2010) de cada sitio (figura 2A), con las dimensiones del árbol y de la competencia, lo que significó menor desarrollo de la extensión de la copa (ec), la más alta tasa de crecimiento en altura y menor incidencia de la luz para los árboles por debajo del dosel. Lang et al. (2010) afirman que, bajo una fuerte competencia, los árboles individuales promueven mayores tasas de crecimiento en altura que en crecimiento lateral, lo que resulta en una menor área de proyección horizontal de copa a los niveles más altos de competencia por luz y copas asimétricas.

Menor proporción de copa, según Condé et al. (2013), indica que el fuste explica la mayor parte de la morfometría en relación a la copa, de acuerdo a los presentes resultados $( \pm 70 \%)$. Los autores concluyen así que la variación en la morfología de las plantas puede sufrir cambios a lo largo de su crecimiento y es susceptible a la influencia de factores tales como espaciamiento, el suelo y el clima, la competencia entre las copas y los tratamientos silvícolas.

Las variaciones del formal de copa, grado de esbeltez, índice de amplitud e índice saliente ocurrieron así, de acuerdo con la edad y el grado de competencia. En los árboles jóvenes, el crecimiento en altura es mayor que el crecimiento de las ramas laterales, formando así una copa más cónica (menor valor del formal de copa) en consecuencia, menor capacidad de asimilación fotosintética y una menor producción en volumen. El grado de esbeltez mostró árboles con menor manto de copa, la competencia por el espacio y recurso, de más rápido crecimiento en al- tura que diámetro y que ocupan los estratos de codominante y dominado, por lo tanto, baja vitalidad y eficiencia en espacio de crecimiento.

El índice de amplitud indicó necesidad de raleo y de espacio con el aumento de la altura de los árboles. Por lo tanto, para la misma altura total, alcanza mayor espacio el individuo que tiene el mayor diámetro de la copa (Cunha y Finger 2013). El índice saliente indicó que ese puede ser utilizado como un indicador de espacio requerido por cada árbol, al ser alcanzado un determinado diámetro. Con el valor promedio se puede establecer cual diámetro de copa es necesario para producir individuos con $40 \mathrm{~cm}$ de diámetro y que crecen sin competencia, indicando este estudio que hay un mayor número de árboles (densidad) que puede soportar el bosque y que reduce sus tasas de crecimiento.

Los resultados del incremento en diámetro mostraron que las menores tasas de crecimiento se asocian con los menores diámetros, lo que explica bosques no manejados y puede comprometer la estructura diametral en el futuro del bosque para formar árboles de mayor diámetro y la necesidad de intervenciones silvícolas.

El estudio de los índices morfométricos e índice de competencia indicó que los bosques de araucaria en el sur de Brasil están en competencia, debido a que los árboles tienen una inestabilidad media, copas más cortas y anchas y mayor densidad de individuos por área, interfiriendo en su desarrollo y crecimiento de copa, apoyando la hipótesis de necesidad de las intervenciones silvícolas para perpetuidad de los remanentes de este tipo de bosque. Las relaciones morfométricas, probada con la correlación de Pearson, son importantes en este sentido para las intervenciones de planificación de raleo, lo que demuestra las condiciones estructurales de los bosques a partir de los cambios en las relaciones de la forma y tamaño de las árboles.

Los valores de índice de la competencia en los sitios son evidentes, explicados por las variables morfométricas y tasas de incremento en diámetro, que indican los límites que los valores pueden llegar de manera que los árboles mantienen las condiciones de crecimiento.

Las correlaciones significativas entre los índices morfométricos explican su importancia para la planificación del manejo forestal, muestran la necesidad de un espacio adecuado para el crecimiento de los árboles, y la inclusión de estos índices en los modelos de crecimiento. A su vez, se expone claramente la estrecha relación entre los índices, la competencia, la influencia del sitio y la necesidad de manejar el bosque.

Relación forma-dimensión. La diferencia de nivel y no de inclinación para la relación proporción de copa en función del diámetro se debe a la diferencia de la relación entre estas variables y su heterogeneidad natural, así como a las condiciones en que cada individuo es desarrollado a lo largo de los años (de competencia, la disponibilidad de recursos), que produce un efecto dependiente de la relación forma-dimensión. 
Sanquetta et al. (2014), ajustando ecuaciones del diámetro de la copa en función del diámetro a la altura del pecho, observaron que la variabilidad en el ajuste de las variables ocurre para los árboles con las mayores o menores dimensiones. También se observó, según los mismos autores, que el $p c \%$ sufrió influencias internas de los árboles causadas por el tamaño y las influencias externas, como la competencia entre los vecinos y las condiciones ambientales.

Lang et al. (2010), en un estudio realizado en el bosque subtropical en el este de China, demostraron que los individuos de todas las especies son muy flexibles en respuesta de crecimiento de la copa para el medio ambiente local. Así, los resultados muestran que existe una variación en la proporción de la copa de acuerdo con el diámetro a la altura del pecho, siendo la especie flexible en adaptación en su ambiente local. Cuanto mayor sea el valor de la proporción de copa $(p c \%)$ mayor la productividad del árbol, pues mide su potencial fotosintético y es sensible a los efectos de competencia, disminuyendo la extensión de la copa y, por lo tanto, altamente correlacionado con el crecimiento (Nutto 2001).

Assmann (1970) menciona que se prefieren los árboles con copas estrechas y largas, ya que pueden crecer en espacio reducido, con el mayor número de árboles por unidad de área. Como medida de la eficiencia de la copa el autor empleó el incremento volumétrico o área basal por metro cuadrado de área de copa. La competencia de árboles que compiten también tuvo un impacto positivo significativo en la cantidad de proporción de la copa. El árbol objetivo tenía mayor poder competitivo, mayor diámetro a la altura del pecho en comparación con el diámetro de los competidores y mayor proporción de copa. Esto está de acuerdo con Lang et al. (2010).

Los resultados indican que, a pesar de la naturaleza ontogénica de la especie para modificar la geometría de la copa con el avance de la edad (Seitz 1986), el efecto de la competencia queda reflejado directamente en la extensión de la copa y, por consiguiente, en la proporción de copa. La línea de regresión para cada sitio muestra que al aumentar el tamaño comienza a realizarse la estabilización de la relación de la copa, la simetría completa (Pretzsch y Dieler 2011), lo que significa que el crecimiento y los recursos que los competidores reciben se independizan de su dimensión.

Con base en el ajuste del modelo, la relación formadimensión puede ser un indicador de la necesidad de raleo en el bosque, pues cuanto menor la proporción de la copa menor la tasa de crecimiento, lo que indica que están en competencia, hay poco espacio para el crecimiento y menos capacidad fotosintética en los estratos inferiores del bosque. La relación indica también, que en los sitios de estas regiones los cambios en las condiciones ambientales y la dinámica del bosque contribuyeron en la aceleración del crecimiento y el desarrollo de las dimensiones diamétricas de la población en las últimas décadas (Pretzsch et al. 2014).

La relación para proporción de copa depende de las condiciones en las que el árbol creció en el bosque, pudiéndo- se encontrar árboles de la misma dimensión con extensión de la copa variable. Sin embargo, una mayor densidad y la competencia tienden a disminuir la proporción de copa, lo que significa disminución de la tasa de crecimiento (menor fotosíntesis), por lo que el monitoreo de esta relación sirve como un indicador de la posibilidad de intervenir en el bosque con la eliminación de los individuos para reducir la densidad y promover el crecimiento de los remanentes, según lo recomendado por el manejo forestal.

Los árboles más grandes presentaron una mayor proporción de la copa, mostrando individuos que obtuvieron alta tasa de crecimiento y la capacidad para destacarse de la competencia por los recursos, el espacio y que ya han realizado el autorraleo, constituyendo así el dosel superior. También es una indicación de que en el proceso inicial de la formación de su estructura, las plantas eran portadoras de alta velocidad de aparición y crecimiento temprano, y que tienen prioridad en la utilización de recursos del medio ambiente, por lo tanto, generalmente tienen una ventaja en su uso (Gustafson et al. 2004).

También indica la capacidad competitiva, porque los individuos desarrollarán rápidamente una arquitectura de copa para interceptar la luz, con la rápida colonización del dosel superior y crecimiento en altura, bien como competencia por la radiación solar en el nivel de copa y, para tener éxito, el árbol debe competir por su posición en la estructura vertical de la comunidad (Lang et al. 2010).

Las diferentes características de la proporción de copa por sitio demuestran la dependencia relativa para la limitación de recursos, espacio y cambios en el diámetro a la altura del pecho. Considerando que las prácticas silvícolas afectan directamente el tamaño de la copa, la inclusión de variables de la copa en el proceso de modelado puede proporcionar una manera de entender mejor y predecir los efectos de los tratamientos silvícolas en el fuste y los atributos de las masas forestales (Sprinz y Burkhart 1987).

\section{CONCLUSIONES}

El estudio de las variables morfométricas e índices de competencia demuestra que los remanentes de bosques de araucaria en sitios naturales en el sur de Brasil están en competencia, así como la necesidad de intervenciones silvícolas para estimular el crecimiento de los árboles que se mantienen. La competencia provoca cambios en la morfometría de la copa y se correlaciona con el diámetro a la altura del pecho, el sitio, el nivel de competencia entre los individuos y los recursos ambientales. Los ajustes de estas variables muestran que cambian de acuerdo a las variaciones en la dinámica de la estructura del bosque.

La generación de ecuaciones de regresión hace posible predecir intervenciones silvícolas para ajustar el número de árboles de acuerdo con el aumento de la proporción de la copa, evitando reducción de las tasas de incremento de diámetro. 


\section{AGRADECIMIENTOS}

Los autores agradecen al Consejo Nacional de Desarrollo Científico y Tecnológico, CNPq por el apoyo financiero para la realización del proyecto, llamado universal 14/2013, la Universidad del Estado de Santa Catarina, FAPESC- Fundación de Apoyo de Investigación del Estado de Santa Catarina y los propietarios de los bosques de araucarias por la disponibilidad de la realización del estudio.

\section{REFERENCIAS}

Álvarez CA, JL Stape, PC Sentelhas, JLM Gonçalves, G Parovek. 2013. Köppen's climate classification map for Brazil. Meteorologische Zeitschrift 22(6): 711-728.

Assmann E. 1970. The principles of forest yield study. Oxford, UK. Pergamon Press. $506 \mathrm{p}$.

Condé TM, MLM de Lima, EML de Neto, H Tonini. 2013. Morfometria de quatro espécies florestais em sistemas agroflorestais no município de Porto Velho, Rondônia. Revista Agro@mbiente 7(1): 18-27.

Costa EA, CAG Finger, FD Fleig. 2016. Influência da posição social nas relações morfométricas de Araucaria angustifolia. Ciência Florestal 26(1): 225-234.

Costa EA, CAG Finger, TR Cunha. 2013. Influência da posição social e do número de raios na estimativa da área de copa em araucária. Revista Floresta 43(3): 429-438.

Cunha TA, CAG Finger. 2013. Competição assimétrica e o incremento diamétrico de árvores individuais de Cedrela odorata L. na Amazônia Ocidental. Acta Amazonica 43(1): 9-18.

Daniels RF, HE Burkhart, TR Clason. 1986. A comparison of competition measures for predicting growth of LoblollyPine trees. Canadian Journal of Forest Research 16(6): $1230-1237$.

Diez JJ. 2012. Sustainable Forest Management - Case Studies. Consultado 30 set. 2015. Disponible en: http://www.intechopen.com/books/sustainable-forest-management-casestudies

EMBRAPA (Empresa Brasileira de Pesquisa Agropecuária, BR). 2012. Sistema brasileiro de classificação dos solos. Brasília. $418 \mathrm{p}$.

Giraudo AR, H Povedano, MJ Belgrano, E Krauczuk, U Pardiñas, A Miquelarena, D Ligier, D Baldo, M Castelino. 2003. Biodiversity status of the interior Atlantic forest of Argentina. In Galindo-Leal C, V Câmara eds. The Atlantic Forest of South America: Biodiversity Status, Threats, and Outlook. Washington DC, USA. Island Press. p. 160-180.

Gustafson DJ, DJ Gibson, DL Nickrent. 2004. Competitive relationships of Andropogon gerardii (Big Bluestem) from remnant and restored native populations and select cultivated varieties. Functional Ecology 18(3): 451-457.

Hess AF, M Minatti, L Ferrari, BA Pintro. 2014. Manejo de Floresta Ombrófila Mista pelo método de Liocourt, Município de Painel, SC. Cerne 20(4): 575-580.

Lang AC, W Härdtle, H Bruelheide, C Geibler, K Nadrowski, A Schuldt, M Yu, G von Oheimb. 2010. Tree morphology responds to neighbourhood competition and slope in species-rich forests of subtropical China. Forest Ecology and Management 260: 1708-1715.

Leite LP, A Zubizarreta-Gerendiain, A Robinson. 2012. Modeling mensurational relationships of plantation - growth loblolly pine (Pinus taeda L.) in Urugay. Forest Ecology and Management 289: 455-462. http://dx.doi.org/10.1016/j. foreco.2012.10.016

McIntosh ACS, AN Gray, SL Garman. 2012. Estimating canopy cover from standard Forest Inventory Measurements in Western Oregon. Forest Science 58(2): 154-167.

Minatti M. 2015. Dendrocronologia aplicada na geração de modelos de crescimento biométricos e ambientais para Araucaria angustifolia na fitorregião do Planalto Serrano, SC. Dissertação de Mestrado. Lages, Brasil. Universidade do Estado de Santa Catarina. $121 \mathrm{p}$.

Nutto L. 2001. Manejo do crescimento diamétrico de Araucaria angustifolia (Bert.) O. Ktze. baseado na árvore individual. Ciência Florestal 11(2): 9-25.

Pretzsch H, G Schütze. 2005. Crown allometry and growing efficiency of Norway spruce (Picea abies (L.) Karst.) and European beech (Fagus sylvatica L.) in pure and mixed stands. Plant Biology 7(6): 628-639.

Pretzsch H, J Dieler. 2011. The dependency of the size-growth relationship of Norway spruce (Picea abies [L.] Karst.) and European beech (Fagus sylvatica [L.]) in forest stands on long-term site conditions, drought events, and ozone stress. Trees 25(3): 355-369.

Pretzsch H, P Biber, G Schütze, TUE Rotzer. 2014. Forest stand growth dynamics in Central Europe have accelerated since 1870. Nature Communications 5, Article ID: 4967. DOI: 10.1038/ncomms5967.

Sanquetta CR, A Behling, APD Corte, AC Fernandes, SM Beckert, AA Simon. 2014. Equações para estimativa do diâmetro de copa para Acácia-negra. Floresta e Ambiente 21(2): 192-205.

Schneider PR, PSP Schneider, CAM de Souza. 2009. Análise de regressão aplicada à Engenharia Florestal. Santa Maria, Brasil. FACOS. 249 p.

Seitz R A. 1986. Crown development of Araucaria angustifolia in its natural environment during sixty years. In Fujimori T, D Whitehead eds. Crown and canopy structure in relation to productivity, Proceedings, Forestry and Forest Products Research Institute. Ibaraki, Japan. p. 129-146.

Stefenon VM, O Gailing, R Finkeldey. 2008. Genetic structure and the conservation of genetic resources of Brazilian pine (Araucaria angustifolia). Forest Ecology and Management 255: 2718-2725.

Sprinz PT, HE Burkhart. 1987. Relationships between tree crown, stem, and stand characteristics in unthinned loblolly pine plantations. Canadian Journal of Forest Research 17(6): 534-538. 
